\title{
Synchronization for a Class of Uncertain Fractional Order Chaotic Systems with Unknown Parameters Using a Robust Adaptive Sliding Mode Controller
}

\author{
Yan Yan \\ Department of Mathematics and Physics, North China Electric Power University, 619 Yonghua North Street, Baoding, \\ Hebei 071003, China \\ Correspondence should be addressed to Yan Yan; yan-yan@ncepu.edu.cn
}

Received 10 March 2016; Revised 16 May 2016; Accepted 30 May 2016

Academic Editor: Riccardo Caponetto

Copyright (C) 2016 Yan Yan. This is an open access article distributed under the Creative Commons Attribution License, which permits unrestricted use, distribution, and reproduction in any medium, provided the original work is properly cited.

This paper deals with the synchronization of a class of fractional order chaotic systems with unknown parameters and external disturbance. Based on the Lyapunov stability theory, a fractional order sliding mode is constructed and a controller is proposed to realize chaos synchronization. The presented method not only realizes the synchronization of the considered chaotic systems but also enhances the robustness of sliding mode synchronization. Finally, some simulation results demonstrate the effectiveness and robustness of the proposed method.

\section{Introduction}

Fractional calculus is as old as conventional calculus and with more than 300 years' history, but its application to physics and engineering is in recent years. It has been found that many systems can be described by fractional order differential equations, for example, in interdisciplinary fields, such as viscoelastic [1], diffusion [2], dynamo theory [3], dengue fever [4], and chemical processing [5], and in nonlinear physical fields such as fractional order Chen system [6], fractional order Lorenz system [7], fractional order unified system [8], fractional Chua circuit [9], fractional order Van der Pol-like oscillator [10], and fractional Newton-Leipnik system [11-14].

Since the pioneering work of Pecora and Carroll [15], chaos synchronization has become a hot topic to the researchers in different fields $[16,17]$. Recently, due to the wide application, many different control methods have been applied to synchronize the fractional order chaotic systems, such as active control [18], adaptive control [19], observerbased control [20], and impulsive control [21]. As the uncertainties are not avoided in the real world, they may lead a given system to an unanticipated state and even destroy the synchronization. Therefore, it is very necessary to investigate robust synchronization to counteract the influence of the uncertainties. Sliding mode control (SMC) is an efficient method to deal with the robust control scheme as it has desired performance such as stability, disturbance rejection capability, and tracking ability. In recent years, sliding mode control method has been applied in the synchronization of fractional order chaotic systems. For example, Tavazoei and Haeri [22] proposed an active SMC to synchronize fractional order chaotic systems. Yin et al. [23] design a SMC to control a class of fractional order chaotic systems. Based on the fractional order line systems' stability theory, Wang et al. [24] proposed an active sliding mode surface and design a controller to realize the modified projective synchronization for two different fractional order systems. In our previous work [25], a novel robust fractional order sliding mode approach for the synchronization of two fractional order chaotic systems in the presence of system parameter uncertain and external disturbance is proposed, but the unknown parameters were not considered. Although Zhang and Yang [26] considered the uncertain master system with unknown parameters and external disturbance, the slave system's external disturbance was not discussed. 
Motivated by the aforementioned analysis, in this paper, we construct a robust synchronization of a class of uncertain fractional chaotic systems via adaptive sliding model control. Based on the designed fractional order integral type sliding surface, an adaptation algorithm is proposed to realize the synchronization of fractional order chaotic systems with unknown parameters, even the fractional order master and slave chaotic system with external disturbance. The numerical simulations show the effectiveness of the proposed method. This paper is organized as follows. In Section 2, the preliminary and system description are presented. Based on the designed fractional sliding mode surface, a robust adaptive controller is proposed to synchronize the class of fractional order chaotic systems in Section 3. A numerical simulation is given in Section 4 to illustrate the effectiveness of the proposed controller. Conclusions are drawn in Section 5.

\section{Preliminary and System Description}

Although fractional calculus is very important in modern science, it has no uniform definition up till now. There are many fractional calculus definitions and among them RiemannLiouville and Caputo definitions are more important than others. As the constant's fractional derivative is zero and its Laplace translation has the traditional initial value, the Caputo definition is used in this paper:

$$
{ }_{0} D_{t}^{\alpha} x(t)=J^{(m-\alpha)} x^{(m)}(t) \quad \text { with } \alpha \in(0,1),
$$

where $m=[\alpha]$; that is, $m$ is the biggest integer which is not less than $\alpha, x^{(m)}$ is the $m$ th-order derivative in the usual sense, and $J^{\beta}(\beta>0)$ is the $\beta$-order fractional order operator with expression

$$
J^{\beta} y(t)=\frac{1}{\Gamma(\beta)} \int_{0}^{t}(t-\tau)^{\beta-1} y(\tau) d \tau,
$$

where $\Gamma(\cdot)$ stands for Euler Gamma function. $D^{\alpha}$ is the shorthand for ${ }_{0} D_{t}^{\alpha}$ in this paper.

Consider a class of fractional order chaotic systems with unknown parameters [23], which is described by

$$
\begin{aligned}
D^{q_{1}} x_{1}= & x_{2} f\left(x_{1}, x_{2}, x_{3}\right)+x_{3} \xi\left(x_{1}, x_{2}, x_{3}\right)-\alpha x_{1} \\
& +d_{1}^{m}(t), \\
D^{q_{2}} x_{2}= & g\left(x_{1}, x_{2}, x_{3}\right)-\beta x_{2}+d_{2}^{m}(t), \\
D^{q_{3}} x_{3}= & x_{2} h\left(x_{1}, x_{2}, x_{3}\right)-x_{1} \xi\left(x_{1}, x_{2}, x_{3}\right)-\gamma x_{3} \\
& +d_{3}^{m}(t),
\end{aligned}
$$

where $x_{1}, x_{2}, x_{3}$ are the states of the system, $f(\cdot), g(\cdot), h(\cdot), \xi(\cdot)$ are smooth nonlinear function of states $x_{1}, x_{2}, x_{3}$, which belong to $R^{3} \rightarrow R$ space, $f(x), g(x), h(x), \xi(x)$ will be used as a shorthand for $f\left(x_{1}, x_{2}, x_{3}\right), g\left(x_{1}, x_{2}, x_{3}\right), h\left(x_{1}, x_{2}, x_{3}\right)$, $\xi\left(x_{1}, x_{2}, x_{3}\right), \alpha, \beta, \gamma$ are the unknown parameters, and $d_{i}(t)$, $i=1,2,3$, is the system's external disturbance.

Remark 1. Note that many fractional order chaotic systems belong to the class characterized by (3) in [23]; examples include the fractional order financial system, the fractional order unified chaotic system (including the fractional order Lorenz system, the fractional order Chen system, and the fractional order Lü system), and the fractional Liu system.

\section{Main Results}

Let system (3) be the drive system, and the response system with a controller is given by

$$
\begin{aligned}
D^{q_{1}} y_{1}= & y_{2} f\left(y_{1}, y_{2}, y_{3}\right)+y_{3} \xi\left(y_{1}, y_{2}, y_{3}\right)-a y_{1} \\
& +d_{1}^{s}(t)+u_{1}(t) \\
D^{q_{2}} y_{2}= & g\left(y_{1}, y_{2}, y_{3}\right)-b y_{2}+d_{2}^{s}(t)+u_{2}(t), \\
D^{q_{3}} y_{3}= & y_{2} h\left(y_{1}, y_{2}, y_{3}\right)-y_{1} \xi\left(y_{1}, y_{2}, y_{3}\right)-c y_{3} \\
& +d_{3}^{s}(t)+u_{3}(t)
\end{aligned}
$$

where $y_{1}, y_{2}, y_{3}$ are the slave system's states, $a, b, c$ are unknown parameters, $d_{i}^{s}, i=1,2,3$, is unknown disturbance, and $u_{i}(t), i=1,2,3$, is designed controller.

The aim in this paper is that, for different initial conditions of systems (3) and (4), the two systems can be synchronized by designing an appropriate control $u(t)$ such that

$$
\lim _{t \rightarrow \infty}\|\mathbf{x}-\mathbf{y}\|=0
$$

where $\mathbf{y}=\left(y_{1}, y_{2}, y_{3}\right), \mathbf{x}=\left(x_{1}, x_{2}, x_{3}\right)$ are state vectors.

Assumption 2. It is assumed that the external disturbances are norm-bounded; that is,

$$
\begin{gathered}
\left\|d_{i}^{m}(t)\right\|<\beta_{i}^{m}, \\
\left\|d_{i}^{s}(t)\right\|<\beta_{i}^{s},
\end{gathered}
$$

$$
i=1,2,3 \text {, }
$$

and $\beta_{i}^{m}+\beta_{i}^{s} \leq \beta_{i}, \quad i=1,2,3$, is satisfied.

The error between the driver system (3) and the slave system (4) can be defined as $\mathbf{e}(t)=\mathbf{x}(t)-\mathbf{y}(t)$. Then the error dynamics is obtained as follows:

$$
\begin{aligned}
D^{q_{1}} \mathbf{e}_{1}= & x_{2} f(\mathbf{x})+x_{3} \xi(\mathbf{x})-\alpha x_{1}+d_{1}^{m}(t)-y_{2} f(\mathbf{y}) \\
& -y_{3} \xi(\mathbf{y})+a y_{1}-d_{1}^{s}(t)-u_{1}(t), \\
D^{q_{2}} \mathbf{e}_{2}= & g(\mathbf{x})-\beta x_{2}+d_{2}^{m}(t)-g(\mathbf{y})+b y_{2}-d_{2}^{s}(t) \\
& -u_{2}(t), \\
D^{q_{3}} \mathbf{e}_{3}= & x_{2} h(\mathbf{x})-x_{1} \xi(\mathbf{x})-\gamma x_{3}+d_{3}^{m}(t)-y_{2} h(\mathbf{y}) \\
& +y_{1} \xi(\mathbf{y})+c y_{3}-d_{3}^{s}(t)-u_{3}(t) .
\end{aligned}
$$

The first step is to select an appropriate sliding mode surface with the desired behavior:

$$
s_{i}(t)=D^{q_{i}-1} \lambda_{i} e_{i}(t), \quad i=1,2,3,
$$

where $\lambda_{i}, i=1,2,3$. 
To ensure the existence of the sliding motion, a discontinuous control law is proposed as

$$
\begin{aligned}
u_{1}(t)= & x_{2} f(\mathbf{x})+x_{3} \xi(\mathbf{x})-\widehat{\alpha} x_{1}+\widehat{\beta}_{1} \operatorname{sgn}\left(s_{1}\right) \\
& -y_{2} f(\mathbf{y})-y_{3} \xi(\mathbf{y})+\widehat{a} y_{1}+k_{1} \operatorname{sgn}\left(s_{1}\right), \\
u_{2}(t)= & g(\mathbf{x})-\widehat{\beta} x_{2}+\widehat{\beta}_{2} \operatorname{sgn}\left(s_{2}\right)-g(\mathbf{y})+\widehat{b} y_{2} \\
& +k_{2} \operatorname{sgn}\left(s_{2}\right), \\
u_{3}(t)= & x_{2} h(\mathbf{x})-x_{1} \xi(\mathbf{x})-\widehat{\gamma} x_{3}+\widehat{\beta}_{3} \operatorname{sgn}\left(s_{3}\right) \\
& -y_{2} h(\mathbf{y})+y_{1} \xi(\mathbf{y})+\widehat{c} y_{3}+k_{3} \operatorname{sgn}\left(s_{3}\right),
\end{aligned}
$$

where $\widehat{\alpha}, \widehat{\beta}, \widehat{\gamma}, \widehat{a}, \widehat{b}, \widehat{c}$, and $\widehat{\gamma}_{i}$ are estimations for $\alpha, \beta, \gamma, a, b, c$, and $\gamma_{i}, i=1,2,3$, respectively.

The fractional order error system is changed into the following formation:

$$
\begin{aligned}
D^{q_{1}} e_{1}= & (\widehat{\alpha}-\alpha) x_{1}+(a-\widehat{a}) y_{1}+d_{1}^{m}(t)-d_{1}^{s}(t) \\
& -\widehat{\beta}_{1} \operatorname{sgn}\left(s_{1}\right)-k_{1} \operatorname{sgn}\left(s_{1}\right), \\
D^{q_{2}} e_{2}= & (\widehat{\beta}-\beta) x_{2}+(b-\widehat{b}) y_{2}+d_{2}^{m}(t)-d_{2}^{s}(t) \\
& -\widehat{\beta}_{2} \operatorname{sgn}\left(s_{2}\right)-k_{2} \operatorname{sgn}\left(s_{2}\right), \\
D^{q_{3}} e_{3}= & (\widehat{\gamma}-\gamma) x_{3}+(c-\widehat{c}) y_{3}+d_{3}^{m}(t)-d_{3}^{s}(t) \\
& -\widehat{\beta}_{3} \operatorname{sgn}\left(s_{3}\right)-k_{3} \operatorname{sgn}\left(s_{3}\right) .
\end{aligned}
$$

The following update laws are defined to tackle the uncertainties, external disturbances, and unknown parameters:

$$
\begin{aligned}
& \dot{\hat{\alpha}}=-x_{1} \lambda_{1} s_{1}, \\
& \dot{\hat{\beta}}=-x_{2} \lambda_{2} s_{2}, \\
& \dot{\hat{\gamma}}=-x_{3} \lambda_{3} s_{3}, \\
& \dot{\hat{a}}=y_{1} \lambda_{1} s_{1}, \\
& \dot{\hat{b}}=y_{2} \lambda_{2} s_{2}, \\
& \dot{\hat{c}}=y_{3} \lambda_{3} s_{3}, \\
& \dot{\hat{\beta}}=\lambda_{i}\left|s_{i}\right| .
\end{aligned}
$$

Theorem 3. If the controller is selected as (9) and the update laws of the unknown parameters are selected as (11), then systems (3) and (4) can be synchronized.

Proof. Selecting a positive definite function as a Lyapunov function candidate

$$
\begin{aligned}
V(t)= & \frac{1}{2} \sum_{i=1}^{3}\left[s_{i}^{2}+\left(\widehat{\beta}_{i}-\beta_{i}\right)^{2}\right]+\frac{1}{2}(\widehat{\alpha}-\alpha)^{2} \\
& +\frac{1}{2}(\widehat{\beta}-\beta)^{2}+\frac{1}{2}(\widehat{\gamma}-\gamma)^{2}+\frac{1}{2}(\widehat{a}-a)^{2} \\
& +\frac{1}{2}(\widehat{b}-b)^{2}+\frac{1}{2}(\widehat{c}-c)^{2},
\end{aligned}
$$

taking its derivative with respect to time $t$, one can get

$$
\begin{aligned}
\dot{V}(t)= & \sum_{i=1}^{3}\left[s_{i} \dot{s}_{i}+\left(\widehat{\beta}_{i}-\beta_{i}\right) \dot{\hat{\beta}}_{i}\right]+(\widehat{\alpha}-\alpha) \dot{\hat{\alpha}} \\
& +(\widehat{\beta}-\beta) \dot{\hat{\beta}}+(\widehat{\gamma}-\gamma) \dot{\hat{\gamma}}+(\widehat{a}-a) \dot{\hat{a}} \\
& +(\widehat{b}-b) \dot{\widehat{b}}+(\widehat{c}-c) \dot{\hat{c}} .
\end{aligned}
$$

Introducing the sliding motion (8) into the right side of (13), one obtains

$$
\begin{aligned}
\dot{V}(t)= & \sum_{i=1}^{3}\left[s_{i} \lambda_{i} D^{q_{i}} e_{i}(t)+\left(\widehat{\beta}_{i}-\beta_{i}\right) \dot{\hat{\beta}}\right]+(\widehat{\alpha}-\alpha) \dot{\hat{\alpha}} \\
& +(\widehat{\beta}-\beta) \dot{\hat{\beta}}+(\widehat{\gamma}-\gamma) \dot{\hat{\gamma}}+(\widehat{a}-a) \dot{\hat{a}} \\
& +(\widehat{b}-b) \dot{\hat{b}}+(\widehat{c}-c) \dot{\hat{c}} .
\end{aligned}
$$

Substituting $D^{q_{i}} e_{i}$ from (10) into (14), this yields

$$
\begin{aligned}
\dot{V}( & t)=s_{1} \lambda_{1}\left[(\widehat{\alpha}-\alpha) x_{1}+d_{1}^{m}(t)-d_{1}^{s}(t)-(\widehat{a}-a) y_{1}\right. \\
& \left.-\widehat{\beta}_{1} \operatorname{sgn}\left(s_{1}\right)\right]-k_{1} \operatorname{sgn}\left(s_{1}\right)+s_{2} \lambda_{2}\left[(\widehat{\beta}-\beta) x_{2}\right. \\
& +d_{2}^{m}(t)-d_{2}^{s}(t)-\widehat{\beta}_{2} \operatorname{sgn}\left(s_{2}\right)-(\widehat{b}-b) y_{2} \\
& \left.-k_{2} \operatorname{sgn}\left(s_{2}\right)\right]+s_{3} \lambda_{3}\left[(\widehat{\gamma}-\gamma) x_{3}+d_{3}^{m}(t)-d_{3}^{s}(t)\right. \\
& \left.-\widehat{\beta}_{3} \operatorname{sgn}\left(s_{3}\right)-k_{3} \operatorname{sgn}\left(s_{3}\right)-(\widehat{c}-c) y_{3}\right] \\
& +\sum_{i=1}^{3}\left(\widehat{\beta}_{i}-\beta_{i}\right) \dot{\hat{\beta}}_{i}+(\widehat{\alpha}-\alpha) \dot{\hat{\alpha}}+(\widehat{\beta}-\beta) \dot{\hat{\beta}}+(\widehat{\gamma}-\gamma) \\
& +\dot{\hat{\gamma}}+(\widehat{a}-a) \dot{\hat{a}}+(\widehat{b}-b) \dot{\hat{b}}+(\widehat{c}-c) \dot{\hat{c} .}
\end{aligned}
$$

Assorting to the update laws (11), (15) is changed into the following forms:

$\dot{V}(t)$

$$
\begin{aligned}
= & s_{1} \lambda_{1}\left[d_{1}^{m}(t)-d_{1}^{s}(t)-\beta_{1} \operatorname{sgn}\left(s_{1}\right)-k_{1} \operatorname{sgn}\left(s_{1}\right)\right] \\
& +s_{2} \lambda_{2}\left[d_{2}^{m}(t)-d_{2}^{s}(t)-k_{2} \operatorname{sgn}\left(s_{2}\right)-\beta_{2} \operatorname{sgn}\left(s_{2}\right)\right] \\
& +s_{3} \lambda_{3}\left[d_{3}^{m}(t)-d_{s}^{3}(t)-k_{3} \operatorname{sgn}\left(s_{3}\right)-\beta_{3} \operatorname{sgn} s_{3}\right] \\
\leq & \left|s_{1}\right| \lambda_{1}\left|d_{1}^{m}(t)-d_{1}^{s}(\mathrm{t})\right|-\beta_{1} \lambda_{1}\left|s_{1}\right|-k_{1} \lambda_{1}\left|s_{1}\right| \\
& +\left|s_{2}\right| \lambda_{2}\left|d_{2}^{m}(t)-d_{2}^{s}(t)\right|-\beta_{2} \lambda_{2}\left|s_{2}\right|-k_{2} \lambda_{2}\left|s_{2}\right| \\
& +\left|s_{3}\right| \lambda_{3}\left|d_{3}^{m}(t)-d_{3}^{s}(t)\right|-\beta_{3} \lambda_{3}\left|s_{3}\right|-k_{3} \lambda_{3}\left|s_{3}\right| \\
\leq & -\lambda_{1} k_{1}\left|s_{1}\right|-\lambda_{2} k_{2}\left|s_{2}\right|-\lambda_{3} k_{3}\left|s_{3}\right|<0 .
\end{aligned}
$$

Using Lyapunov stability theory, it can be concluded that the drive system (3) and the slave system (4) realize the synchronization. 


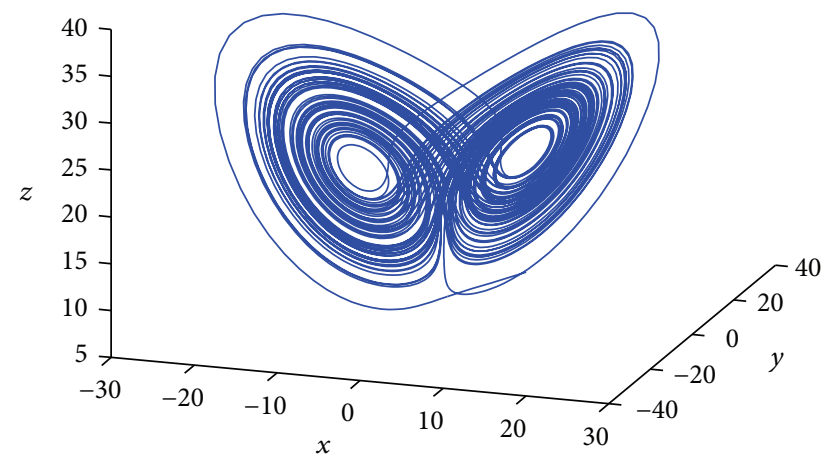

FIGURE 1: 2.89-order fractional Chen system.

\section{Simulation}

In this section, two numerical simulations are presented to show the efficiency of the proposed method.

Example 4. Consider the fractional order Chen system [6] which is written as

$$
\begin{aligned}
& D^{q_{1}} x_{1}=a_{1}\left(x_{2}-x_{1}\right), \\
& D^{q_{2}} x_{2}=d_{1} x_{1}-x_{1} x_{3}+c_{1} x_{2}, \\
& D^{q_{3}} x_{3}=x_{1} x_{2}-b_{1} x_{3},
\end{aligned}
$$

where $\left(a_{1}, b_{1}, c_{1}, d_{1}\right)=(35,-3,28,-7)$. The system is chaotic with $q_{1}=0.98, q_{2}=0.96, q_{3}=0.95$ and initial value $(10,0,10)$ and its chaotic attractor is shown in Figure 1.

Regarding (3) and (4), the drive and slave systems are given as follows:

$$
\begin{aligned}
& D^{q_{1}} x_{1}=x_{2} \alpha-\alpha x_{1}+0.5 \cos t, \\
& D^{q_{2}} x_{2}=d x_{1}-\beta x_{2}+0.5 \sin 2 t, \\
& D^{q_{3}} x_{3}=x_{2} x_{1}-\gamma x_{3}+0.5 \cos 3 t, \\
& D^{q_{1}} y_{1}=y_{2} a-a y_{1}+0.5 \sin t+u_{1}(t), \\
& D^{q_{2}} y_{2}=d y_{1}-y_{1} y_{3}-b y_{2}+0.5 \cos 2 t+u_{2}(t), \\
& D^{q_{3}} x_{3}=y_{2} y_{1}-c y_{3}+0.5 \sin 3 t+u_{3}(t) .
\end{aligned}
$$

The discontinuous control law corresponding to (9) is

$$
\begin{aligned}
u_{1}(t)= & x_{2} \alpha-\widehat{\alpha} x_{1}+\widehat{\beta} \operatorname{sgn}\left(s_{1}\right)-y_{2} a+\widehat{a} y_{1} \\
& +k_{1} \operatorname{sgn}\left(s_{1}\right), \\
u_{2}(t)= & d x_{1}-x_{1} x_{3}-\widehat{\beta}_{2} x_{2}+\widehat{\beta}_{2} \operatorname{sgn}\left(s_{2}\right)-d y_{1} \\
& +y_{1} y_{3}+\widehat{b} y_{2}+k_{2} \operatorname{sgn}\left(s_{2}\right), \\
u_{3}(t)= & x_{2} x_{1}-\widehat{\gamma} x_{3}+\widehat{\beta}_{3} \operatorname{sgn}\left(s_{3}\right)-y_{2} y_{1}+\widehat{c} y_{3} \\
& +k_{3} \operatorname{sgn}\left(s_{3}\right)
\end{aligned}
$$

with the sliding mode surface (8) and update laws (11); $d=-7$ and the systems are started with initial values $\left(x_{1}, x_{2}, x_{3}\right)=$
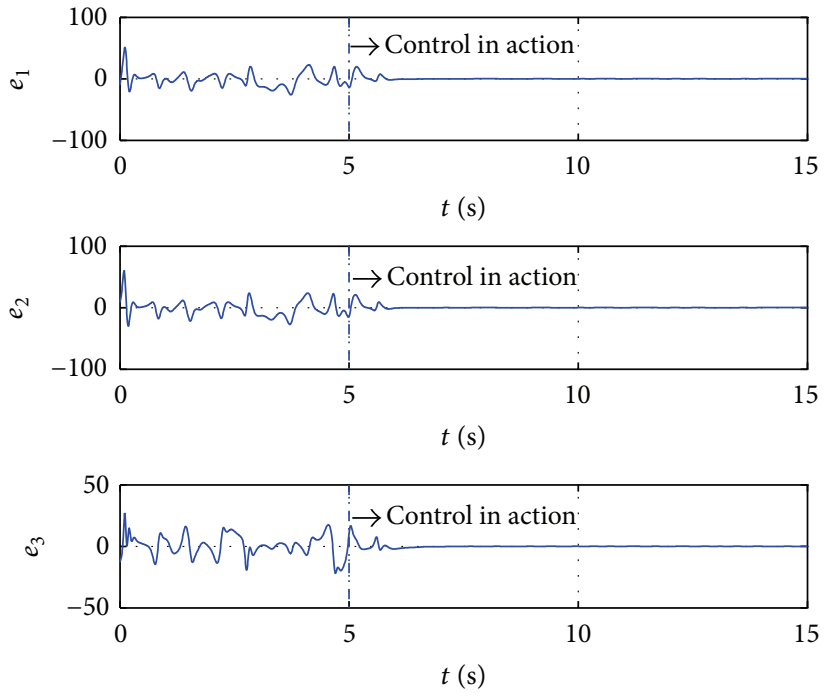

FIGURE 2: Synchronization errors of the drive and slave of fractional Chen systems.
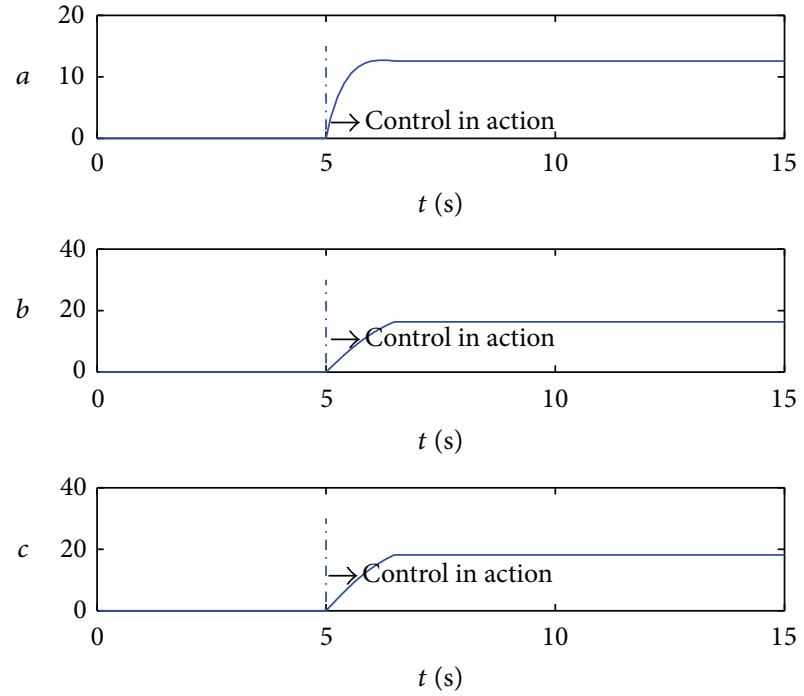

FIgURE 3: Time response of the update parameters $a, b, c$ of fractional Chen system.

$(9,10,1),\left(y_{1}, y_{2}, y_{3}\right)=(10,0,10)$; then the simulation results are shown in Figures 2, 3, 4, and 5. Figure 2 illustrates the synchronization errors of the drive and slave systems decrease to 0 , where the control inputs are turned on at $t=5 \mathrm{~s}$. It can been seen that the chaos synchronization between the drive system and slave system is realized. The time responses of the update vector parameters are depicted in Figures 3-5, respectively.

Example 5. Consider the fractional order Lorenz system [7], which is expressed as

$$
\begin{aligned}
& D^{q_{1}} x_{1}=a_{1}\left(x_{2}-x_{1}\right), \\
& D^{q_{2}} x_{2}=x_{1}\left(b_{1}-x_{3}\right)-x_{2},
\end{aligned}
$$


$\alpha$
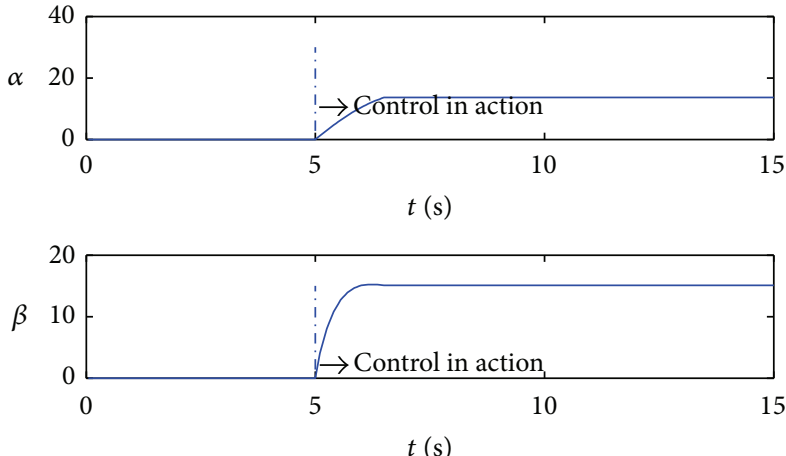

$t(\mathrm{~s})$

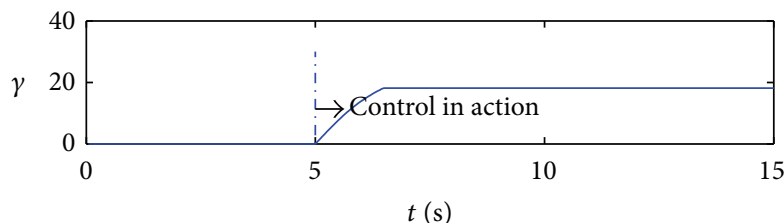

FIgURE 4: Time response of the update parameters $\alpha, \beta, \gamma$ of fractional Chen system.

$$
D^{q_{3}} x_{3}=x_{1} x_{2}-c_{1} x_{3}
$$

where $\left(a_{1}, b_{1}, c_{1}\right)=(10,28,8 / 3)$. The system exhibits a chaotic behavior as shown in Figure 6 with $q_{1}=q_{2}=q_{3}=0.99$ and initial value $(10,0,10)$. Regarding (3) and (4), the drive and slave systems are given as follows:

$$
\begin{aligned}
& D^{q_{1}} x_{1}=\alpha\left(x_{2}-x_{1}\right)+0.5 \cos t, \\
& D^{q_{2}} x_{2}=x_{1}\left(\beta-x_{3}\right)-x_{2}+0.5 \sin 2 t, \\
& D^{q_{3}} x_{3}=x_{1} x_{2}-\gamma x_{3}+0.5 \cos 3 t, \\
& D^{q_{1}} x_{1}=a\left(x_{2}-x_{1}\right)+0.5 \sin t+u_{1}(t), \\
& D^{q_{2}} x_{2}=x_{1}\left(b-x_{3}\right)-x_{2}+0.5 \cos 2 t+u_{2}(t), \\
& D^{q_{3}} x_{3}=x_{1} x_{2}-c x_{3}+0.5 \sin 3 t+u_{3}(t) .
\end{aligned}
$$

The discontinuous control law corresponding to (9) is

$$
\begin{aligned}
u_{1}(t)= & x_{2} \alpha-\widehat{\alpha} x_{1}+\widehat{\beta}_{1} \operatorname{sgn}\left(s_{1}\right)-y_{2} a+\widehat{a} y_{1} \\
& +k_{1} \operatorname{sgn}\left(s_{1}\right), \\
u_{2}(t)= & x_{1}\left(\beta-x_{3}\right)-\widehat{\beta} x_{2}+\widehat{\beta}_{2} \operatorname{sgn}\left(s_{2}\right)-y_{1}\left(b-y_{3}\right) \\
& +\widehat{b} y_{2}+k_{2} \operatorname{sgn}\left(s_{2}\right), \\
u_{3}(t)= & x_{1} x_{2}-\widehat{\gamma} x_{3}+\widehat{\beta}_{3} \operatorname{sgn}\left(s_{3}\right)-y_{2} y_{1}+\widehat{c} y_{3} \\
& +k_{3} \operatorname{sgn}\left(s_{3}\right)
\end{aligned}
$$

with the sliding mode surface (8), update laws (11), and initial values $\left(x_{1}, x_{2}, x_{3}\right)=(8,-2,10),\left(y_{1}, y_{2}, y_{3}\right)=(-7,10,-5)$; then the simulation results are shown in Figures 7-10. Figure 7 illustrates the synchronization errors of the drive

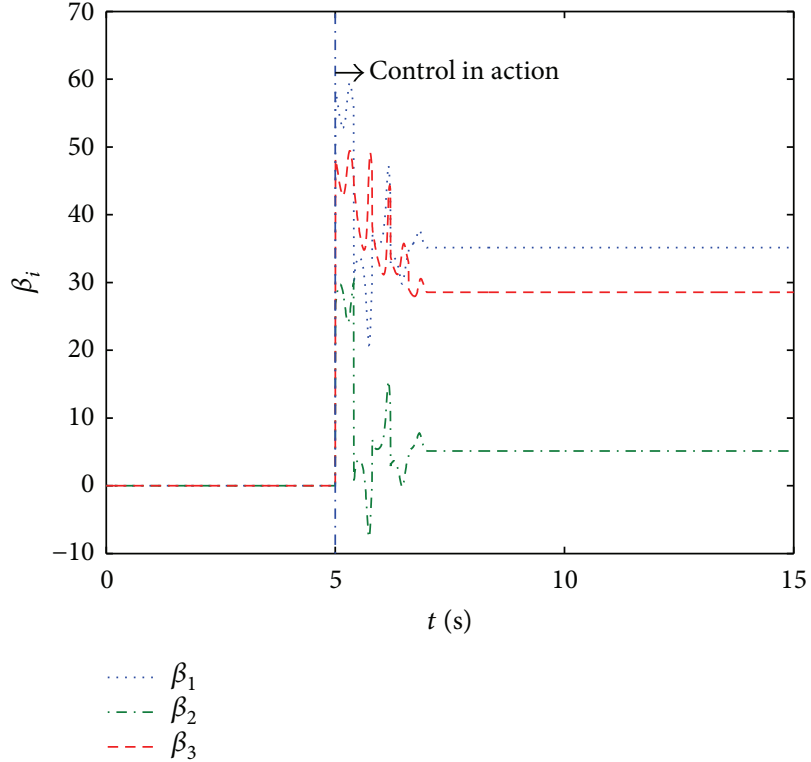

FIgURE 5: Time response of the update parameter $\beta_{i}, i=1,2,3$, of fractional Chen system.

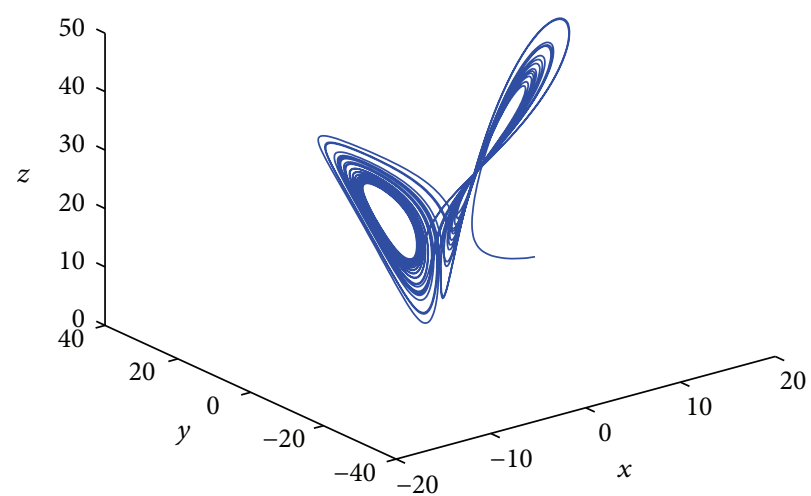

FIGURE 6: 2.97-order fractional Lorenz system.
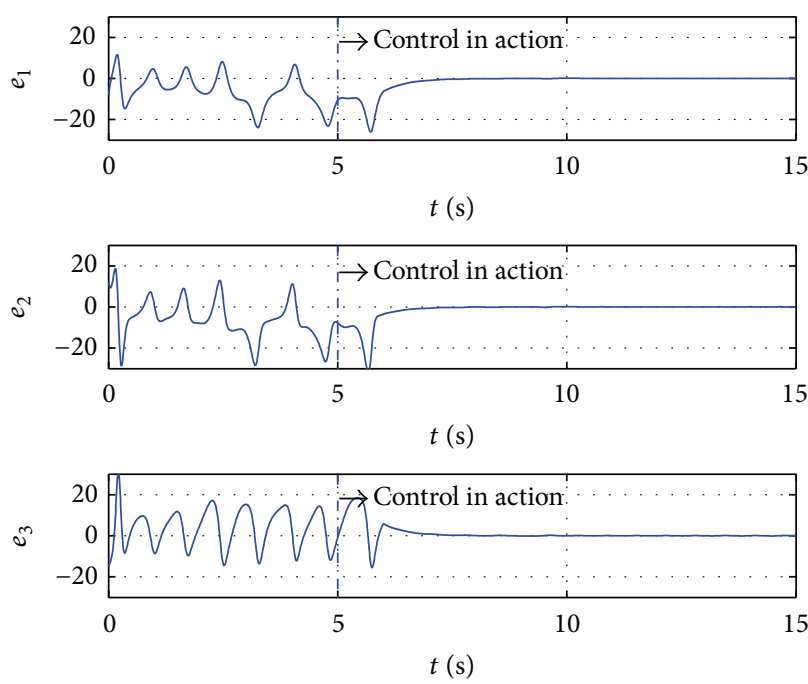

FIGURE 7: Synchronization errors of the drive and slave of fractional Lorenz systems. 

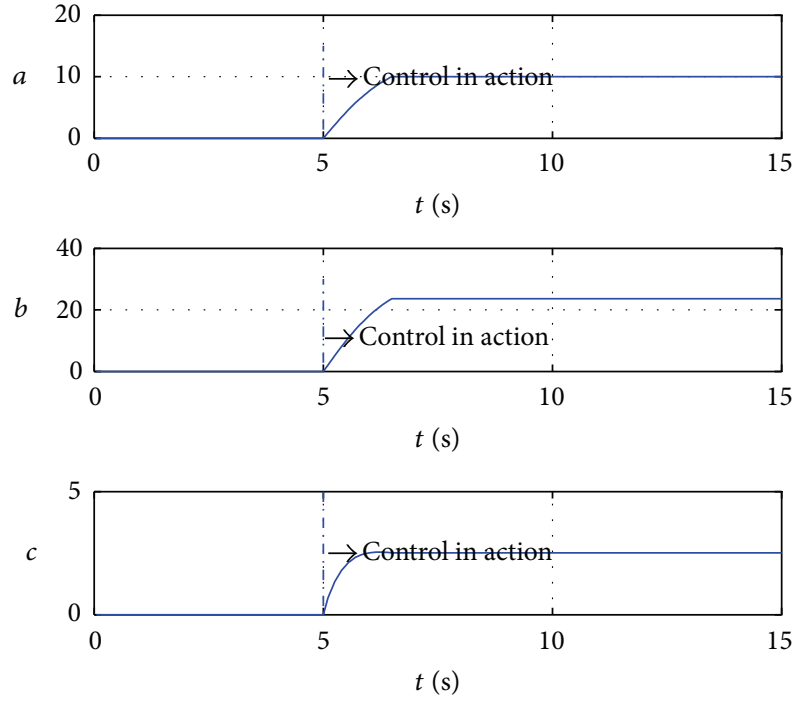

FIGURE 8: Time response of the update parameters $a, b, c$ of fractional Lorenz system.
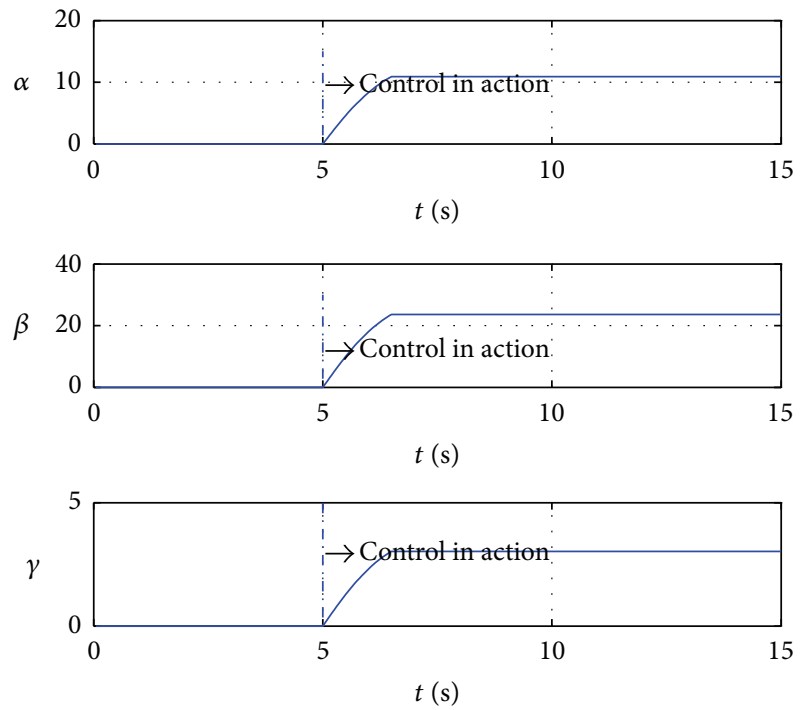

FIgURE 9: Time response of the update parameters $\alpha, \beta, \gamma$ of fractional Lorenz system.

and slave systems decrease to 0 , where the control inputs are turned on at $t=5 \mathrm{~s}$. It can been seen that the chaos synchronization between the drive system and slave system is also realized. The time responses of the update vector parameters are depicted in Figures 8-10, respectively.

\section{Conclusions}

In this paper, a robust adaptive sliding mode controller has been designed to synchronize a class of uncertain fractional chaotic systems with unknown parameters. Based on the Lyapunov stability theory, the designed closed-loop system is stable and the proposed robust adaptive controller can realize chaotic systems' synchronization. Finally, two numerical

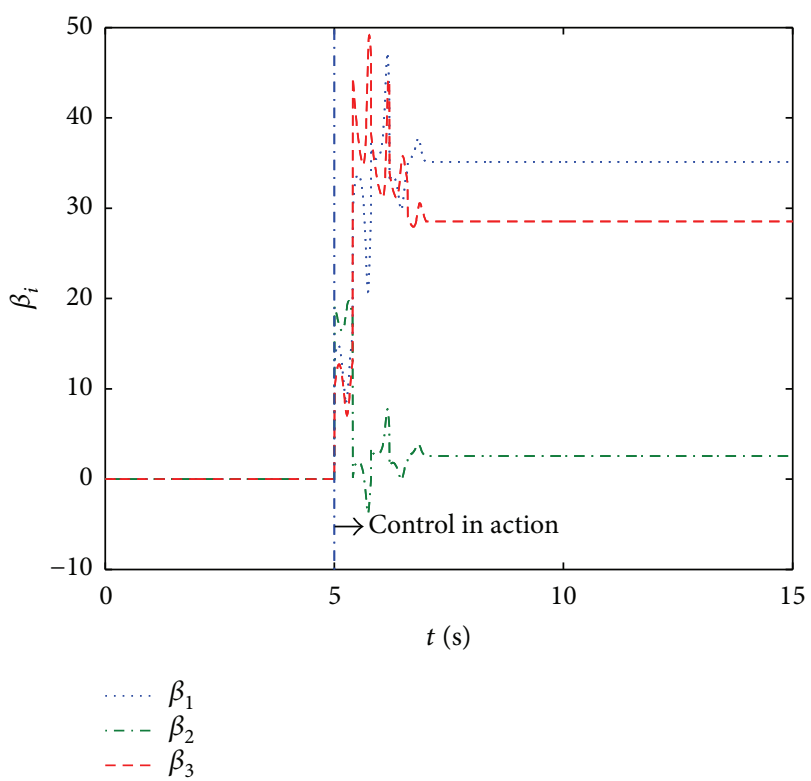

FIgURE 10: Time response of the update parameter $\beta_{i}, i=1,2,3$, of fractional Lorenz system.

examples have been shown to demonstrate the effectiveness of the proposed scheme.

\section{Competing Interests}

The authors declared that they have no conflict of interests regarding the publication of this work. The authors declare that they do not have any commercial or associative interest that represents a conflict of interests in connection with the work.

\section{Acknowledgments}

This research was supported by the National Nature Science Foundation of Hebei Province under Project no. F2016502025, the Fundamental Research Funds for the Central Universities under Project no. 9161015007, and the National Nature Science Foundation of China under Project no. 61403137.

\section{References}

[1] M. El-Shahed, "MHD of a fractional viscoelastic fluid in a circular tube," Mechanics Research Communications, vol. 33, no. 2, pp. 261-268, 2006.

[2] I. S. Jesus and J. T. Machado, "Fractional control of heat diffusion systems," Nonlinear Dynamics, vol. 54, no. 3, pp. 263282, 2008.

[3] P. Muthukumar, P. Balasubramaniam, and K. Ratnavelu, "Synchronization of a novel fractional order stretch-twist-fold (STF) flow chaotic system and its application to a new authenticated encryption scheme (AES)," Nonlinear Dynamics, vol. 77, no. 4, pp. 1547-1559, 2014.

[4] K. Diethelm, "A fractional calculus based model for the simulation of an outbreak of dengue fever," Nonlinear Dynamics, vol. 71, no. 4, pp. 613-619, 2013. 
[5] A. Flores-Tlacuahuac and L. T. Biegler, "Optimization of fractional order dynamic chemical processing systems," Industrial \& Engineering Chemistry Research, vol. 53, no. 13, pp. 5110-5127, 2014.

[6] W. Deng and C. Li, "Synchronization of chaotic fractional Chen system," Journal of the Physical Society of Japan, vol. 74, no. 6, pp. 1645-1648, 2005.

[7] I. Grigorenko and E. Grigorenko, "Chaotic dynamics of the fractional Lorenz system," Physical Review Letters, vol. 91, no. 3, Article ID 034101, 4 pages, 2003.

[8] W. Deng and C. Li, "The evolution of chaotic dynamics for fractional unified system," Physics Letters A, vol. 372, no. 4, pp. 401-407, 2008

[9] T. T. Hartley, C. F. Lorenzo, and H. K. Qammer, "Chaos in a fractional order Chua's system," IEEE Transactions on Circuits and Systems I: Fundamental Theory and Applications, vol. 42, no. 8, pp. 485-490, 1995.

[10] M. Attari, M. Haeri, and M. S. Tavazoei, "Analysis of a fractional order van der pol-like oscillator via describing function method," Nonlinear Dynamics, vol. 61, no. 1-2, pp. 265-274, 2010.

[11] L.-J. Sheu, H.-K. Chen, J.-H. Chen et al., "Chaos in the newton-Leipnik system with fractional order," Chaos, Solitons \& Fractals, vol. 36, no. 1, pp. 98-103, 2008.

[12] G. M. Mahmoud, T. M. Abed-Elhameed, and M. E. Ahmed, "Generalization of combination-combination synchronization of chaotic n-dimensional fractional-order dynamical systems," Nonlinear Dynamics, vol. 83, no. 4, pp. 1885-1893, 2016.

[13] S. Shao, M. Chen, and X. Yan, "Adaptive sliding mode synchronization for a class of fractional-order chaotic systems with disturbance," Nonlinear Dynamics, vol. 83, no. 4, pp. 1855-1866, 2016.

[14] P. Zhou and R. Bai, "The adaptive synchronization of fractionalorder chaotic system with fractional-order $1<q<2$ via linear parameter update law," Nonlinear Dynamics, vol. 80, no. 1-2, pp. 753-765, 2015.

[15] L. M. Pecora and T. L. Carroll, "Synchronization in chaotic systems," Physical Review Letters, vol. 64, no. 8, pp. 821-824, 1990.

[16] H. Zhang, D. Liu, and Z. Wang, Controlling Chaos: Suppression, Synchronization and Chaotification, Springer, 2009.

[17] H. Zhang, T. Ma, G.-B. Huang, and Z. Wang, "Robust global exponential synchronization of uncertain chaotic delayed neural networks via dual-stage impulsive control," IEEE Transactions on Systems, Man, and Cybernetics, Part B: Cybernetics, vol. 40, no. 3, pp. 831-844, 2010.

[18] M. Srivastava, S. P. Ansari, S. K. Agrawal, S. Das, and A. Y. Leung, "Anti-synchronization between identical and nonidentical fractional-order chaotic systems using active control method," Nonlinear Dynamics, vol. 76, no. 2, pp. 905-914, 2014.

[19] S. K. Agrawal and S. Das, "A modified adaptive control method for synchronization of some fractional chaotic systems with unknown parameters," Nonlinear Dynamics, vol. 73, no. 1-2, pp. 907-919, 2013.

[20] D. M. Senejohnny and H. Delavari, "Active sliding observer scheme based fractional chaos synchronization," Communications in Nonlinear Science and Numerical Simulation, vol. 17, no. 11, pp. 4373-4383, 2012.

[21] I. Stamova, "Global Mittag-Leffler stability and synchronization of impulsive fractional-order neural networks with timevarying delays," Nonlinear Dynamics, vol. 77, no. 4, pp. 12511260, 2014.
[22] M. S. Tavazoei and M. Haeri, "Synchronization of chaotic fractional-order systems via active sliding mode controller," Physica A: Statistical Mechanics and Its Applications, vol. 387, no. 1, pp. 57-70, 2008.

[23] C. Yin, S.-M. Zhong, and W.-F. Chen, "Design of sliding mode controller for a class of fractional-order chaotic systems," Communications in Nonlinear Science and Numerical Simulation, vol. 17, no. 1, pp. 356-366, 2012.

[24] X. Wang, X. Zhang, and C. Ma, "Modified projective synchronization of fractional-order chaotic systems via active sliding mode control," Nonlinear Dynamics, vol. 69, no. 1-2, pp. 511-517, 2012.

[25] L. Zhang and Y. Yan, "Robust synchronization of two different uncertain fractional-order chaotic systems via adaptive sliding mode control," Nonlinear Dynamics, vol. 76, no. 3, pp. 1761-1767, 2014.

[26] R. Zhang and S. Yang, "Robust synchronization of two different fractional-order chaotic systems with unknown parameters using adaptive sliding mode approach," Nonlinear Dynamics, vol. 71, no. 1-2, pp. 269-278, 2013. 


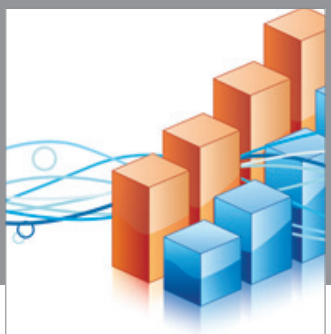

Advances in

Operations Research

vatem alat4

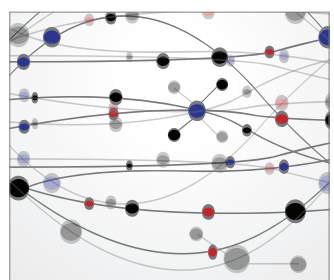

\section{The Scientific} World Journal
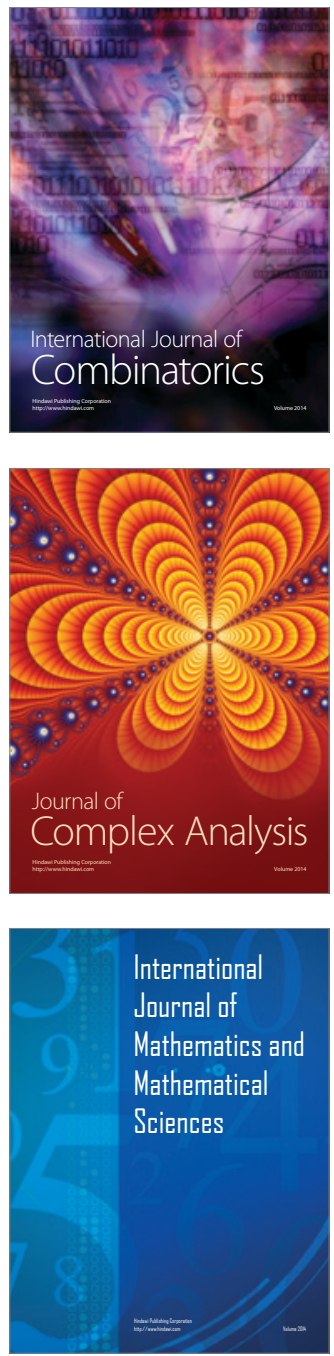
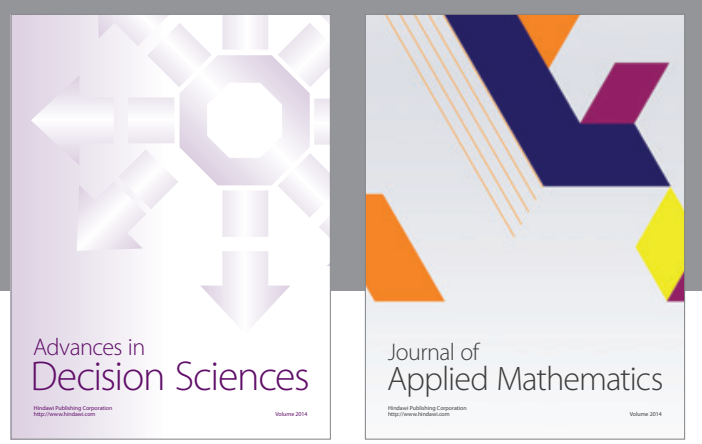

Algebra

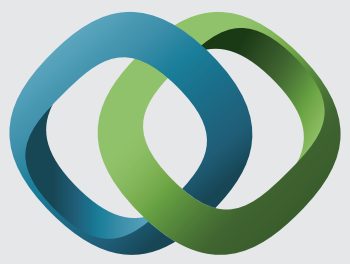

\section{Hindawi}

Submit your manuscripts at

http://www.hindawi.com
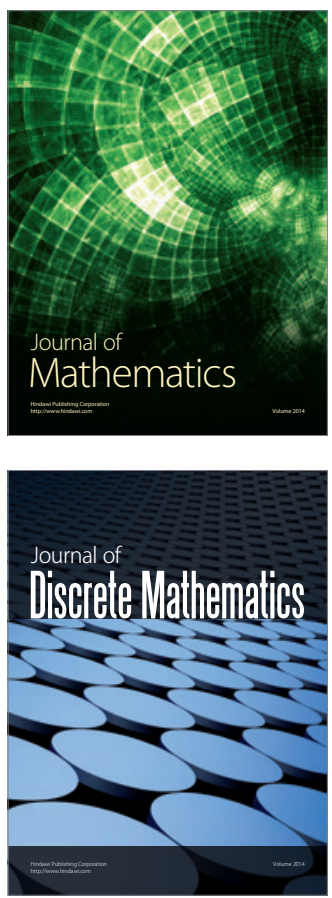

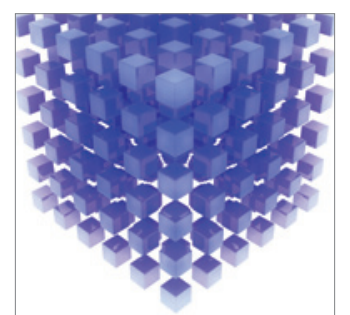

Mathematical Problems in Engineering
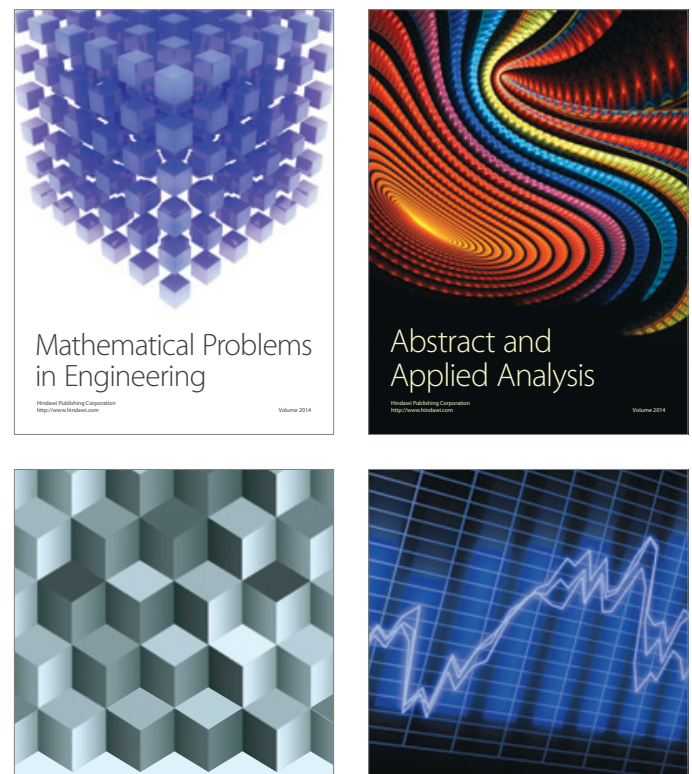

Journal of

Function Spaces

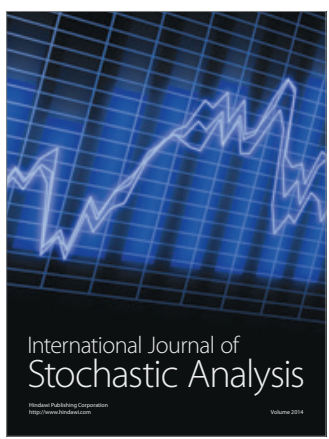

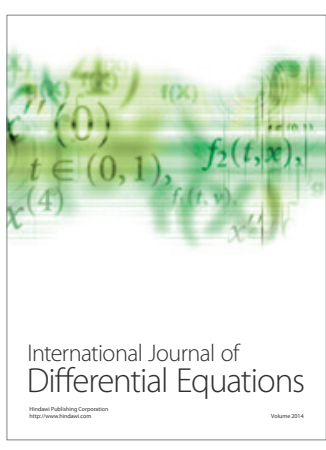
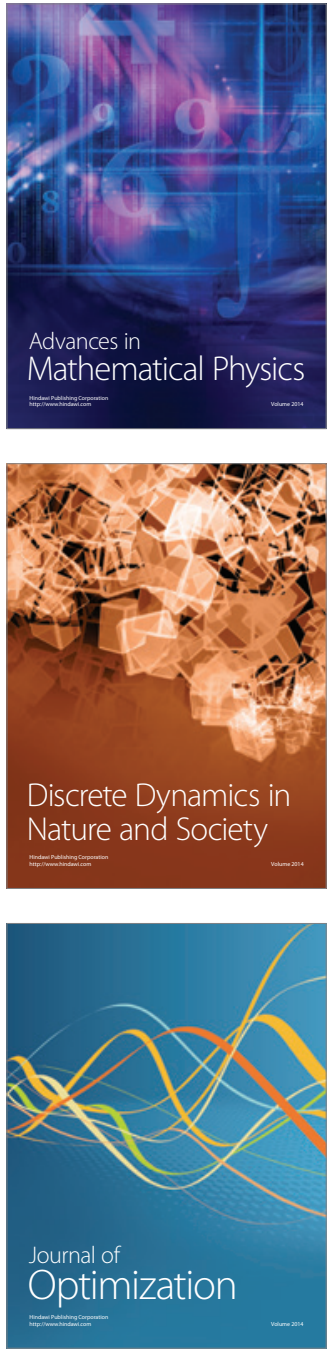